\title{
Comparison of Eleven Anthropometric Indices for the Screening for Non-Alcoholic Fatty Liver Disease in Patients with Diabetes
}

\section{Xintong Guo}

Guangxi Medical University First Affiliated Hospital

Min Liang ( $\square$ liangm@gxmu.edu.cn )

Guangxi Medical University First Affiliated Hospital https://orcid.org/0000-0002-8354-1093

\section{Research}

Keywords: Metabolic syndrome, lipid accumulation product, Non-alcoholic fatty liver disease

Posted Date: July 1st, 2021

DOl: https://doi.org/10.21203/rs.3.rs-649055/v1

License: (c) (1) This work is licensed under a Creative Commons Attribution 4.0 International License.

Read Full License 


\section{Abstract}

Background \& Aims

Nonalcoholic fatty liver disease (NAFLD) is considered as the main cause of chronic liver disease, so the prediction of NAFLD in high-risk cohort of patients would be valuable. Although evidence indicates that anthropometric clinical indicators has been a worthy choice in the prediction of NAFLD, there is no consensus on which is the best obesity indexes for the identification of NAFLD in high-risk patients. This study aimed to compare the screening ability for NAFLD of eleven anthropometric indices in patients with diabetes.

Methods

The sample included 905 type 2 diabetic patients diagnosed by US. We selected 11 formulas: triponderal mass index (TMI), triglyceride glucose index (TyG), a body shape index (ABSI), Clínica Universidad de Navarra-body adiposity estimator (CUN-BAE), lipid accumulation product (LAP), visceral adiposity index (VAI), Hepatic Steatosis Index (HIS), Body Mass Index (BMI), conicity index (Cl), Waist to Height ratio (WHtR), and Body Roundness Index (BRI), and calculated their performance of NAFLD screening.

Results

The percentage of US reductions were, 49.55 (VAl), 58.70 (TyG), 50.70 (TMI), 54.80 (LAP), 67.10 (HIS), 32.40 (CUN-BAE), 28.24 (Cl), 48.30 (BRI), 33.28 (ABSI), 43.57 (BMI), 48.30 (WHtR). The false negative percentage were 10.29 (VAI), 18.81 (TyG), 14.49 (TMI), 11.28 (LAP), 21.02 (HIS), 8.74 (CUN-BAE), 6.08 (Cl), 9.96 (BRI), 9.62 (ABSI), 11.28 (BMI), 9.96 (WHtR).

Conclusion

Taking negative predictive value (NPV), positive predictive value (PPV), and other aspects into account, the best formula to use for NAFLD screening was LAP.

\section{Introduction}

Nonalcoholic fatty liver disease (NAFLD) has become the most common liver disease globally with a prevalence of $24 \%{ }^{1}$. Patients with NAFLD can progress to non-alcoholic steatohepatitis (NASH) that could cause cirrhosis and the onset of hepatocarcinoma ${ }^{2,3}$. Besides liver diseases, NAFLD can also lead to metabolic syndrome (MetS), type 2 diabetes (T2DM), arteriosclerotic cardiovascular disease and high incidence of colorectal tumors. NAFLD is very common in patients with T2DM. It is estimated that about $62 \%$, or two-thirds of type 2 diabetes are hepatic steatosis ${ }^{4}$. Compared with patients with NAFLD, patients with both T2DM and NAFLD had an increased risk of (NASH), hepatic fibrosis, cardiovascular disease and diabetic complications, which is associated with a high risk of morbidity related to liver damage severity, imposing early detection to prevent these complications. NAFLD is also closely related to MetS and has also emerged as the largest chronic liver disease in China with the high risk of MetS. 
The obesity-related indicators, of low cost and convenience, have been prevalent in the prediction of NAFLD in recent years ${ }^{5}$. For example, the visceral adiposity index (VAl) and the triglycerides and glucose (TyG) index have been applied in a NAFLD-related prediction models ${ }^{6}$. Besides, body roundness index (BRI), a body shape index (ABSI), conicity index (Cl), Clínica Universidad de Navarra-body adiposity estimator (CUN-BAE), triponderal mass index (TMI) have proved to be effective predictors of MetS and NAFLD ${ }^{7-9}$.

Anthropometric indicators recommended in the literature have already been used for NAFLD prediction ${ }^{10}$, ${ }^{11}$, while none of these studies have evaluated predictive capacity in individuals with T2DM ${ }^{12}$. This study aimed to assess the ability of obesity-related indices to screening NAFLD. Furthermore, we also explored the relationship between NAFLD and MetS.

\section{Material And Methods}

\section{Subjects}

From March 2019 to March 2021, 2,032 patients with diabetes aged 41-95 years (mean $59.36 \pm 7.45$ ) were involved in this study. Participants were hospitalized in the First Affiliated Hospital of Guangxi Medical University. These patients were excluded: (1) patients infected; (2) patients with severe CVDs, heart failure, cancer, thyroid dysfunction, significant renal impairments; (3) type 1 diabetes; (4) alcoholic fatty liver disease (AFLD), active chronic hepatitis B and C, acute forms, autoimmune hepatitis, cirrhosis, hemochromatosis, regular consumption of drugs associated with fatty liver disease. Finally, 905 subjects (379 women and 526 men) were included. The Ethics Committee of the First Affiliated Hospital of Guangxi Medical University approved the study protocol. This study was conducted in conformance with the principles of Helsinki Declaration. All subjects provided informed consent.

\section{Selected formulas}

The calculations of the obesity indices are as follows:

$\mathrm{BMI}=$ weight $(\mathrm{kg}) /$ height $^{2}\left(\mathrm{~m}^{2}\right)$;

WHtR = WC $(\mathrm{cm}) /$ Height $(\mathrm{cm})$;

$\mathrm{Cl}=0.109^{-1} \mathrm{WC}(\mathrm{m})[\text { Weight }(\mathrm{kg}) / \text { Height }(\mathrm{m})]^{-1 / 2}$;

$A B S I=W C(m) / B M I^{2 / 3}(\mathrm{~kg} / \mathrm{m}) \times$ height $^{1 / 213}$;

$\mathrm{BRI}=364.2-365.5\left[1-\pi^{-2} \mathrm{WC}^{2}(\mathrm{~m}) \text { Height }^{-2}(\mathrm{~m})\right]^{1 / 2} ;{ }^{14}$

$\mathrm{TMI}=$ Weight $(\mathrm{kg}) / \operatorname{Height}^{3}(\mathrm{~m})^{15}$.

$\mathrm{HIS}=8 \times(\mathrm{ALT} / \mathrm{AST})+\mathrm{BMI}(+2$, if female $;+2$, if diabetes mellitus $)$ 
$\mathrm{LAP}=\mathrm{WC}(\mathrm{cm})-65 \times \mathrm{TG}(\mathrm{mmol} / \mathrm{L})$ in males $\mathrm{LAP}=\mathrm{WC}(\mathrm{cm})-58 \times \mathrm{TG}(\mathrm{mmol} / \mathrm{L})$ in females

$\mathrm{VAl}=\mathrm{WC}(\mathrm{cm}) /[39.68+(1.88 \times \mathrm{BMI})] \times \mathrm{TG}(\mathrm{mmol} / \mathrm{L}) / 1.03 \times 1.31 / \mathrm{HDL}(\mathrm{mmol} / \mathrm{L})$ in males ;

$\mathrm{VAI}=\mathrm{WC}(\mathrm{cm}) /[36.58+(1.89 \times \mathrm{BMI})] \times \mathrm{TG}(\mathrm{mmol} / \mathrm{L}) / 0.81 \times 1.52 / \mathrm{HDL}(\mathrm{mmol} / \mathrm{L})$ in females.

CUN-BAE $=-44.988+(0.503 \times$ age $)+(10.689 \times$ gender $)+(3.172 \times \mathrm{BMI})-(0.026 \times \mathrm{BMI})+(0.181 \times \mathrm{BMI} \times$ gender $)-(0.02 \mathrm{BMI} \times$ age $)-(0.005 \times \mathrm{BMI} \times$ gender $)+(0.00021 \times \mathrm{BMI} 2 \times$ age $)$ (zero for males and one for females). ${ }^{16}$

TyG $=\ln \left(\right.$ fasting triglycerides $(\mathrm{mg} / \mathrm{dL}) \times$ fasting glucose $(\mathrm{mg} / \mathrm{dL}) / 2^{17}$

\section{Definition of Metabolic Syndrome}

MetS was defined as the guideline of Chinese Diabetes Society. Participants had to meet at least 3 factors: 1) elevated $W C \geq 90 \mathrm{~cm}$ in men, $\geq 85 \mathrm{~cm}$ in women, 2) elevated FPG $\geq 6.1 \mathrm{mmol} / \mathrm{L}$ or diagnosed with diabetes, 3) blood pressure $\geq 130 / 85 \mathrm{mmHg}$ or diagnosed with hypertension, 4) elevated TG $\geq 1.7$ $\mathrm{mmol} / \mathrm{L}$, and 5) reduced $\mathrm{HDL}-\mathrm{C}<1.04 \mathrm{mmol} / \mathrm{L}$

\section{Definition of NAFLD}

NAFLD were defined as ultrasound test evidence of diffuse hepatic steatosis and excludes other causes such as severe alcohol consumption, viral or drug hepatic. The ultrasound examinations were performed by a professional sonographer.

\section{Data Collection}

The homeostasis model assessment of insulin resistance (HOMA-IR) index was calculated by HOMA Calculator v2.2.2. ${ }^{18}$ The smoking and alcohol consumption were collected from medical records. Two categories were considered: drinking/smoking (daily, occasional and former drinking/smoking) and nondrinking/smoking (never drinking/smoking). Hepatitis B antigens and hepatitis C antibodies were examined to exclude viral hepatitis.

\section{Measurements}

Height and weight (with light clothing and shoes removed) were measured using a calibrated weighing scale with a precision of $1 \mathrm{~cm}$ and $1 \mathrm{~kg}$. WC was measured using an un-stretched tape with a precision of $1 \mathrm{~cm}$.

After 10 minutes of rest in a sitting position, blood pressure (BP) (systolic and diastolic blood pressure) was measured for 3 times using a corrected mercury sphygmomanometer. BP was recorded as the average of the three readings.

\section{Biochemical Factors}


All the biochemical markers were originated from medical records. Blood samples were collected from the antebrachial vein after an overnight fast.

\section{Statistical Analysis}

Mean and standard deviation (SD) were reported for continuous variables. Percentage and frequency were reported for discrete variables. one-way analysis of variance (ANOVA) tests was used to Compare normally distributed continuous variables. If one variable is highly skewed, the Manne Whitney test was used to compare the two groups. Proportion were analyzed by Chi-square and Fisher's exact tests. Logistic regression models were used to reflect the correlations with odds ratio (OR) and 95\% confidence interval $(\mathrm{Cl})$. For the evaluation of the prediction ability of different obesity indices, receiver operating characteristic (ROC) analyses were performed. Sensitivity and specificity were calculated applying the optimal cut-off point calculated. All statistical analyses were performed using SPSS version 23.0. The ROC analysis was completed using MedCalc version 12.0. $P$ values less than 0.05 were considered statistically significant.

\section{Results}

Table 1 shows the clinical characteristics of the participants in the NAFLD and NO-NAFLD group. Prevalence of NAFLD was $44.6 \%$, with a higher percentage of females (47.2\%) than males (42.7\%). Participants with NAFLD had higher triglyceride TG, C peptide, IR, SBP, DBP and lower HDL compared to those without NAFLD (all $P<0.05$ ). The female participants with NAFLD had higher AST, ALT, and uric acid compared to those without NAFLD (all $P<0.05$ ). All the anthropometric indexes were significantly higher in the NAFLD than in the NO-NAFLD group. No difference was observed for age, TC, LDL, fasting plasma glucose FPG, and HbA1 in subjects with and without NAFLD.

Table 2 shows that participants with NAFLD had higher prevalence rates of MetS and its components. The overall prevalence rate of MetS was $69.3 \%$ (73.1\% in males; $64 \%$ in females). No difference was observed for smoking and drinking in subjects with and without NAFLD. Figure 1 shows the significant trends of stepwise increases in the prevalence of NAFLD with the increasing sum of components of MetS $(p<0.001)$. The more the sum of components of MetS, the higher prevalence of NAFLD: NO-NAFLD $(86.3 \%, 70.5 \%, 53.5 \%, 44.6 \%$, and $29.7 \%)$; NAFLD $(13.8 \%, 29.5 \%, 46.5 \%, 55.4 \%$, and $70.3 \%)$.

Table 3 shows that the obesity indices included in the study were independently associated with NAFLD, even after adjustment for sex, age, and history of drinking and smoking. The odds ratios grew along with quartiles for almost all analyzed anthropometric indices, except $\mathrm{ABSI}$ and $\mathrm{Cl}$. The highest odds ratios were noted for the following indicators: $\mathrm{BMI}(\mathrm{OR}=9.055,95 \% \mathrm{Cl}$ : 5.777-14.191; $\mathrm{p}<0.001) ;(\mathrm{OR}=11.001$, 95\%Cl: 5.95-20.34; $p<0.001)$; LAP (OR = 9.729, 95\%Cl: 5.607-16.88; $p<0.001)$; and TMI (OR = 8.684, 95\%Cl: 5.55-13.59; $p<0.001)$.

Table 4 shows the ROC analysis of eleven indicators for predicting NAFLD. The Area Under the ROC curve (AUROC) appears ampler in females than in males for all formulas. The ROC analysis showed that, 
except for $\mathrm{Cl}$ and $\mathrm{ABSI}$, all indexes mentioned in the study were able to discriminate between patients with and without NAFLD (AUCs $>0.6, P<0.05$ ). Figure 2 represents the ROC curves for the eleven formulas in different subgroups. In male diabetic patients aged 40-59 years, LAP (0.768) shows the highest AUC; in females aged 40-59 years, BMI (0.713) shows high AUC. For patients over age 60, BRI (0.745) and WHtR (0.745) have the highest AUC values in men and LAP (0.749) in women. WHtR and BRI were similar in ROC results, which may be explained by the same indicators involved in these two formulas. It is evident that CUN-BAE showed the highest odds ratio for NAFLD. However, LAP shows the best performance in AUROC analysis. This may be interpreted by the different statistical methods used. LAP index had the highest Youden index values (0.348). The cut-off value of LAP was 42.

Table 4 also represents PPV, NPV, reduction of US, and the proportion of subjects identified as at risk, patients with unidentified NAFLD (NAFLD missed), patients diagnosed in the high-risk group screened by obesity indices (NAFLD in risk group) and patients confirmed of total diseased.

\section{Discussion}

The NAFLD prevalence in diabetic patients is higher in females than in males. NAFLD group had significantly higher biochemical indexes and obesity indexes suggesting metabolic impairments than the NO-NAFLD group. Only in females, we found hepatic enzymes (ALT/AST) level is higher in NAFLD group than No-NAFLD group, while the mean level in the NAFLD group was not over upper limit of normal (ULN). This result confirmed with the study that demonstrated elevated levels of ALT/AST in 54\% of NAFLD patients ${ }^{19}$.

Our findings confirm that MetS was closely related to NAFLD, and finds a stepwise increase in the prevalence of NAFLD according to the number of components of MetS. Significant differences of the key markers of metabolic dysfunction were also found between NAFLD group and NO-NAFLD group. In the NAFLD group, there were significantly higher prevalence of MetS, lower levels of HDL and higher levels of WC and TG than in NO-NAFLD group, proving conclusion in other studies that most patients with NAFLD had same relevant MetS features ${ }^{20,21}$. Previous studies have reported that as a main cause of MetS, IR plays a critical role in the progress of NAFLD. IR leads to increases in insulin levels, which enhances lipid storage by promoting hepatic triglycerides synthesis and inhibiting lipolysis ${ }^{22}$. This could explain why IR works in the association between MetS and NAFLD. Since extrahepatic complications occur across multiple organ systems, the extra-hepatic impact includes CVD, one feature of MetS, has been the main causes of death in NAFLD patients ${ }^{23}$.

The values of the 11 obesity indices analyzed resulted higher in the NAFLD than the NO-NAFLD group as confirmed in other studies. A previous study showed good discriminatory ability of BMI, WHtR, Cl, LAP, and $A B S I$ were measured in the diagnosis of $\mathrm{NAFLD}^{9}$, and another study reported that TyG level is an independent risk factor of NAFLD, and TyG index plays a partial mediating role in the relationship between WHtR and NAFLD ${ }^{24}$. In patients with type 2 diabetes, the non-invasive HIS score is considered as an easy, convenient and inexpensive screening for fatty liver ${ }^{25}$. This study also demonstrated the 
associations among NAFLD and various obesity-related indices, which further supported for the application of these indices to predict NAFLD. The fact may be explained by the presence of obesity with IR and dyslipidemia, which can promote hepatic steatosis. Furthermore, visceral fat, subcutaneous fat as well as IR could be important in the pathogenesis of NAFLD ${ }^{26-28}$.

For all indices, the calculated AUC showed larger areas for females than males. The largest AUC of these indices were different in different sex and age groups. The best percentage for identifying NAFLD was obtained using $\mathrm{Cl}$. With a view to the false negative rate (NAFLD missed \%), the best performance was achieved by CUN-BAE while HIS was the worst. On the other hand, HIS is the best methods to reduce the need for US, followed by TyG and LAP. On the basis of cut-offs obtained, we compared the real performance of each formula. The results suggest that all the formulas can reduce the number of US. The best performance was obtained with HIS, TyG and LAP, and the worst performance with CUN-BAE, $\mathrm{Cl}$ and ABSI. These findings are in accordance with the PPV of each formula considered.

Our findings recommend LAP the best formula to identify people with NAFLD combined a high PPV and NPV. It combined a good reduction in the need for US, with a small false negative rate (\% NAFLD missed). A cross-sectional study of 40,459 subjects demonstrated that LAP was significantly associated with the severity of NAFLD, and that LAP had a strong diagnostic value for NAFLD ${ }^{29}$. Another study also confirmed that LAP is a strong and easily obtainable predictive index of NAFLD for children ${ }^{30}$. Furthermore, it is simple to apply as was calculated separately in males and females, and it requires only one anthropometric measure and one biochemical index and reflect the body content of visceral fat theoretically.

There are several shortcomings in this study. First, causality cannot be established due to the crosssectional study design. Second, individual-level factors such as economic status, occupation, social activities were not included in our analysis, which may have some extent influence on fatty liver. Third, the study population involving only residents of southern China, further studies involving other populations are therefore warranted to add to the reliability of this finding.

\section{Conclusion}

In this study, relationships among MetS and obesity-related indices with NAFLD have been proved, and the stepwise increases in the prevalence of NAFLD with the components of MetS is further evidence for this conclusion. In this study, LAP was considered as the best indices to identify diabetic patients with NAFLD. Furthermore, it is simple to apply as was calculated separately in males and females, and it requires only one anthropometric measure and one biochemical index. Some anthropometric indicators have already been used for NAFLD prediction, while few researches have been conducted to compare the ability of screening NAFLD of obesity-related indices, especially in high-risk groups. These screening markers for NAFLD may contribute to an early diagnosis of NAFLD and to a timely therapeutic intervention in diabetic patients in a convenient and noninvasive way. 


\section{Abbreviations}

NAFLD: Non-alcoholic fatty liver disease; TMI: triponderal mass index (TMI), TyG: triglyceride glucose index; ABSI: a body shape index; CUN-BAE: Clínica Universidad de Navarra-body adiposity estimator; LAP: lipid accumulation product; VAl: visceral adiposity index; HIS: Hepatic Steatosis Index; BMI: Body Mass Index; Cl: conicity index; WHtR: Waist to Height ratio; BRI: Body Roundness Index; US: Ultrasound Scan; PPV: positive predictive value; NPV: negative predictive value; NASH: non-alcoholic steatohepatitis; T2DM: type 2 diabetes; AUROC: area under the receiver operating characteristic curve; NASH: NonAlcoholic Steatohepatitis; MetS: metabolic syndrome; MRI: Magnetic Resonance Imaging; AFLD: alcoholic fatty liver disease; ALT: serum alanine aminotransferase; HBV: active chronic hepatitis B; HCV: active chronic hepatitis C; SD: standard deviation; ROC: receiver operating characteristic curve; ALT/AST: Alanine amino transferase to aspartate amino transferase ratio; TG: triglycerides;

\section{Declarations}

\section{Acknowledgements}

Not applicable

\section{Author contributions}

Xintong Guo conceived and designed the research, collected samples, analyzed the data and wrote the manuscript. Min Liang revised the manuscript. All authors read and approved the final manuscript.

\section{Funding}

Not applicable.

\section{Availability of data and materials}

The datasets generated and/or analyzed during the current study are publicly available.

\section{Ethics approval and consent to participate}

The Ethics Committee of the First Affiliated Hospital of Guangxi Medical University approved the study protocol. This study was conducted in conformance with the principles of Helsinki Declaration. All subjects provided informed consent.

\section{Consent for publication}

Not applicable.

\section{Competing interests}

The authors declare that they have no competing interests. 


\section{References}

1. Cusi K, Sanyal A, Zhang S, et al. Non-alcoholic fatty liver disease (NAFLD) prevalence and its metabolic associations in patients with type 1 diabetes and type 2 diabetes. Diabetes, obesity \& metabolism. 2017,19(11):1630-1634. doi:10.1111/dom.12973

2. Bifari F, Manfrini R, Dei Cas M, et al. Multiple target tissue effects of GLP-1 analogues on nonalcoholic fatty liver disease (NAFLD) and non-alcoholic steatohepatitis (NASH). Pharmacological research. 2018,137:219-229. doi:10.1016/j.phrs.2018.09.025

3. Nimer N, Choucair I, Wang Z, et al. Bile acids profile, histopathological indices and genetic variants for non-alcoholic fatty liver disease progression. Metabolism: clinical and experimental. 2021,116:154457. doi:10.1016/j.metabol.2020.154457

4. Tilg H, Moschen AR, Roden M. NAFLD and diabetes mellitus. Nature reviews Gastroenterology \& hepatology. Jan 2017,14(1):32-42. doi:10.1038/nrgastro.2016.147

5. Almeida NS, Rocha R, Cotrim HP, Daltro C. Anthropometric indicators of visceral adiposity as predictors of non-alcoholic fatty liver disease: A review. World J Hepatol. Oct 27 2018,10(10):695701. doi:10.4254/wjh.v10.i10.695

6. Cen C, Wang W, Yu S, et al. Development and validation of a clinical and laboratory-based nomogram to predict nonalcoholic fatty liver disease. Hepatology international. 2020,14(5):808-816. doi:10.1007/s12072-020-10065-7

7. Lin I, Lee M, Wang C, Wu D, Chen S. Gender Differences in the Relationships among Metabolic Syndrome and Various Obesity-Related Indices with Nonalcoholic Fatty Liver Disease in a Taiwanese Population. International journal of environmental research and public health. 2021,18(3)doi:10.3390/ijerph18030857

8. Procino F, Misciagna G, Veronese N, et al. Reducing NAFLD-screening time: A comparative study of eight diagnostic methods offering an alternative to ultrasound scans. Liver international : official journal of the International Association for the Study of the Liver. 2019,39(1):187-196. doi:10.1111/liv.13970

9. Zhang Y, Li B, Liu N, Wang P, He J. Evaluation of Different Anthropometric Indicators for Screening for Nonalcoholic Fatty Liver Disease in Elderly Individuals. International journal of endocrinology. 2021,2021:6678755. doi:10.1155/2021/6678755

10. Bedogni G, Bellentani S, Miglioli L, et al. The Fatty Liver Index: a simple and accurate predictor of hepatic steatosis in the general population. BMC gastroenterology. Nov 2 2006,6:33. doi:10.1186/1471-230x-6-33

11. Wang J, Xu C, Xun Y, et al. ZJU index: a novel model for predicting nonalcoholic fatty liver disease in a Chinese population. Sci Rep. Nov 16 2015,5:16494. doi:10.1038/srep16494

12. Cuthbertson DJ, Weickert MO, Lythgoe D, et al. External validation of the fatty liver index and lipid accumulation product indices, using $1 \mathrm{H}$-magnetic resonance spectroscopy, to identify hepatic 
steatosis in healthy controls and obese, insulin-resistant individuals. European journal of endocrinology. Nov 2014,171(5):561-9. doi:10.1530/eje-14-0112

13. Krakauer NY, Krakauer JC. A New Body Shape Index Predicts Mortality Hazard Independently of Body Mass Index. Plos One. Jul 18 2012,7(7)e39504. doi:10.1371/journal.pone.0039504

14. Thomas DM, Bredlau C, Bosy-Westphal A, et al. Relationships Between Body Roundness with Body Fat and Visceral Adipose Tissue Emerging from a New Geometrical Model. Obesity. Nov 2013,21(11):2264-2271. doi:10.1002/oby.20408

15. Peterson CM, Su H, Thomas DM, et al. Tri-Ponderal Mass Index vs Body Mass Index in Estimating Body Fat During Adolescence. Jama Pediatrics. Jul 2017,171(7):629-636. doi:10.1001/jamapediatrics.2017.0460

16. Gomez-Ambrosi J, Silva C, Catalan V, et al. Clinical Usefulness of a New Equation for Estimating Body Fat. Diabetes Care. Feb 2012,35(2):383-388. doi:10.2337/dc11-1334

17. Guerrero-Romero F, Simental-Mendia LE, Gonzalez-Ortiz M, et al. The Product of Triglycerides and Glucose, a Simple Measure of Insulin Sensitivity. Comparison with the Euglycemic-Hyperinsulinemic Clamp. Journal of Clinical Endocrinology \& Metabolism. Jul 2010,95(7):3347-3351. doi:10.1210/jc.2010-0288

18. Geloneze B, Vasques A, Stabe C, et al. HOMA1-IR and HOMA2-IR indexes in identifying insulin resistance and metabolic syndrome: Brazilian Metabolic Syndrome Study (BRAMS). Arquivos brasileiros de endocrinologia e metabologia. 2009,53(2):281-7. doi:10.1590/s000427302009000200020

19. Bedogni G, Miglioli L, Masutti F, Tiribelli C, Marchesini G, Bellentani S. Prevalence of and risk factors for nonalcoholic fatty liver disease: the Dionysos nutrition and liver study. Hepatology (Baltimore, Md). 2005,42(1):44-52. doi:10.1002/hep.20734

20. Muzurović E, Mikhailidis D, Mantzoros C. Non-Alcoholic Fatty Liver Disease, Insulin Resistance, Metabolic Syndrome and their Association with Vascular Risk. Metabolism: clinical and experimental. 2021:154770. doi:10.1016/j.metabol.2021.154770

21. Marchesini G, Bugianesi E, Forlani G, et al. Nonalcoholic fatty liver, steatohepatitis, and the metabolic syndrome. Hepatology. Apr 2003,37(4):917-23. doi:10.1053/jhep.2003.50161

22. Gaggini M, Morelli M, Buzzigoli E, DeFronzo RA, Bugianesi E, Gastaldelli A. Non-alcoholic fatty liver disease (NAFLD) and its connection with insulin resistance, dyslipidemia, atherosclerosis and coronary heart disease. Nutrients. May 10 2013,5(5):1544-60. doi:10.3390/nu5051544

23. Ballestri S, Zona S, Targher G, et al. Nonalcoholic fatty liver disease is associated with an almost twofold increased risk of incident type 2 diabetes and metabolic syndrome. Evidence from a systematic review and meta-analysis. Journal of gastroenterology and hepatology. May 2016,31(5):936-44. doi:10.1111/jgh.13264

24. Huanan C, Sangsang L, Amoah A, et al. Relationship between triglyceride glucose index and the incidence of non-alcoholic fatty liver disease in the elderly: a retrospective cohort study in China. BMJ open. 2020,10(11):e039804. doi:10.1136/bmjopen-2020-039804 
25. Fennoun $\mathrm{H}$, Mansouri S, Tahiri M, et al. Interest of hepatic steatosis index (HSI) in screening for metabolic steatopathy in patients with type 2 diabetes. The Pan African medical journal. 2020,37:270. doi:10.11604/pamj.2020.37.270.9087

26. Hsiao TJ, Chen JC, Wang JD. Insulin resistance and ferritin as major determinants of nonalcoholic fatty liver disease in apparently healthy obese patients. International journal of obesity and related metabolic disorders : journal of the International Association for the Study of Obesity. Jan 2004,28(1):167-72. doi:10.1038/sj.ijo.0802519

27. Milić S, Lulić D, Štimac D. Non-alcoholic fatty liver disease and obesity: biochemical, metabolic and clinical presentations. World journal of gastroenterology. Jul 28 2014,20(28):9330-7. doi:10.3748/wjg.v20.i28.9330

28. Thamer C, Machann J, Haap M, et al. Intrahepatic lipids are predicted by visceral adipose tissue mass in healthy subjects. Diabetes care. Nov 2004,27(11):2726-9. doi:10.2337/diacare.27.11.2726

29. Dai H, Wang W, Chen R, Chen Z, Lu Y, Yuan H. Lipid accumulation product is a powerful tool to predict non-alcoholic fatty liver disease in Chinese adults. Nutrition \& metabolism. 2017,14:49. doi:10.1186/s12986-017-0206-2

30. Özcabı B, Demirhan S, Akyol M, Öztürkmen Akay H, Güven A. Lipid accumulation product is a predictor of nonalcoholic fatty liver disease in childhood obesity. Korean journal of pediatrics. Dec 2019,62(12):450-455. doi:10.3345/kjp.2019.00248

\section{Tables}

Due to technical limitations, tables are only available as a download in the Supplemental Files section.

\section{Figures}




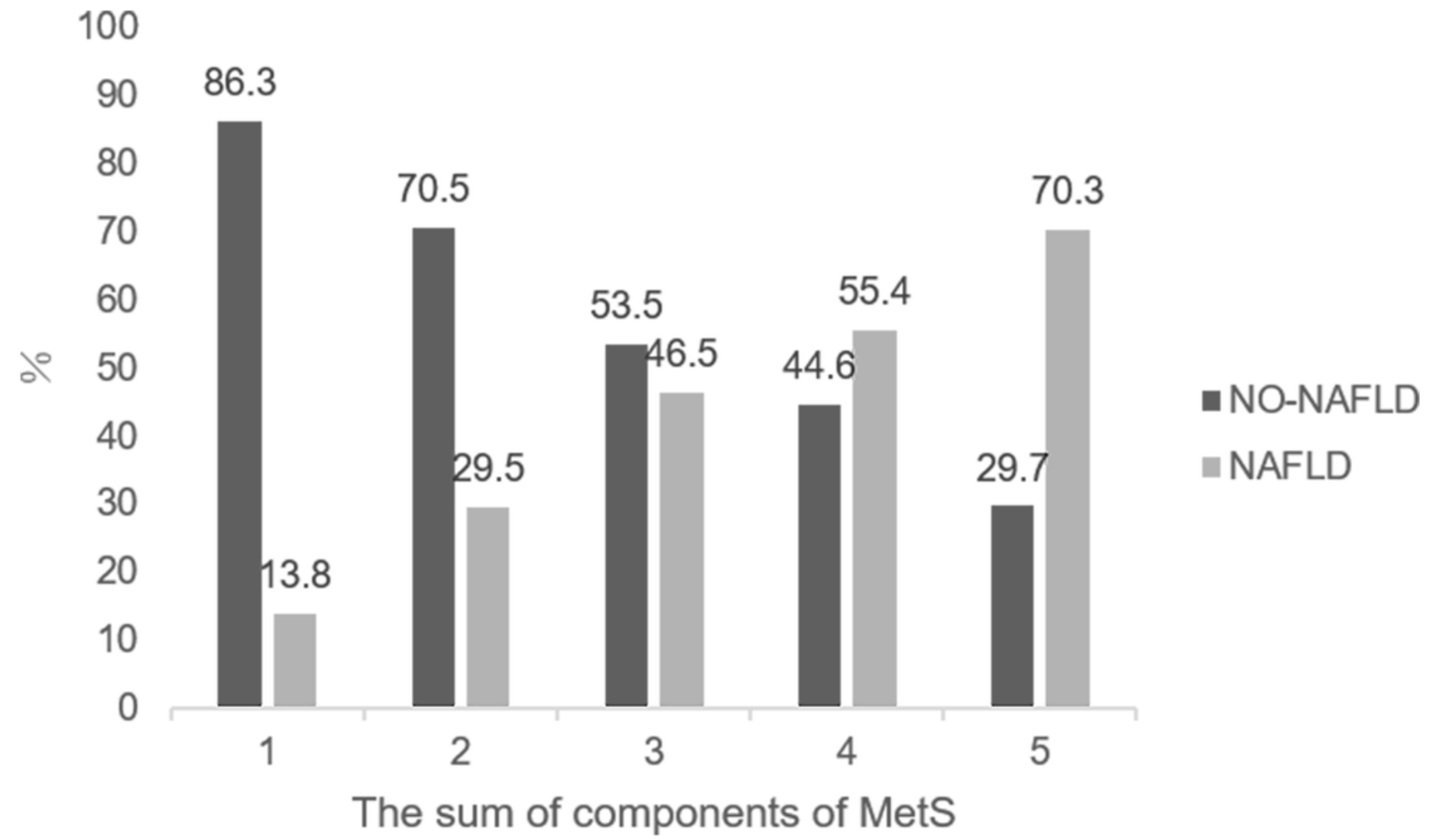

\section{Figure 1}

The trends for stepwise increases in the prevalence of NAFLD corresponding to the sum of components of MetS 

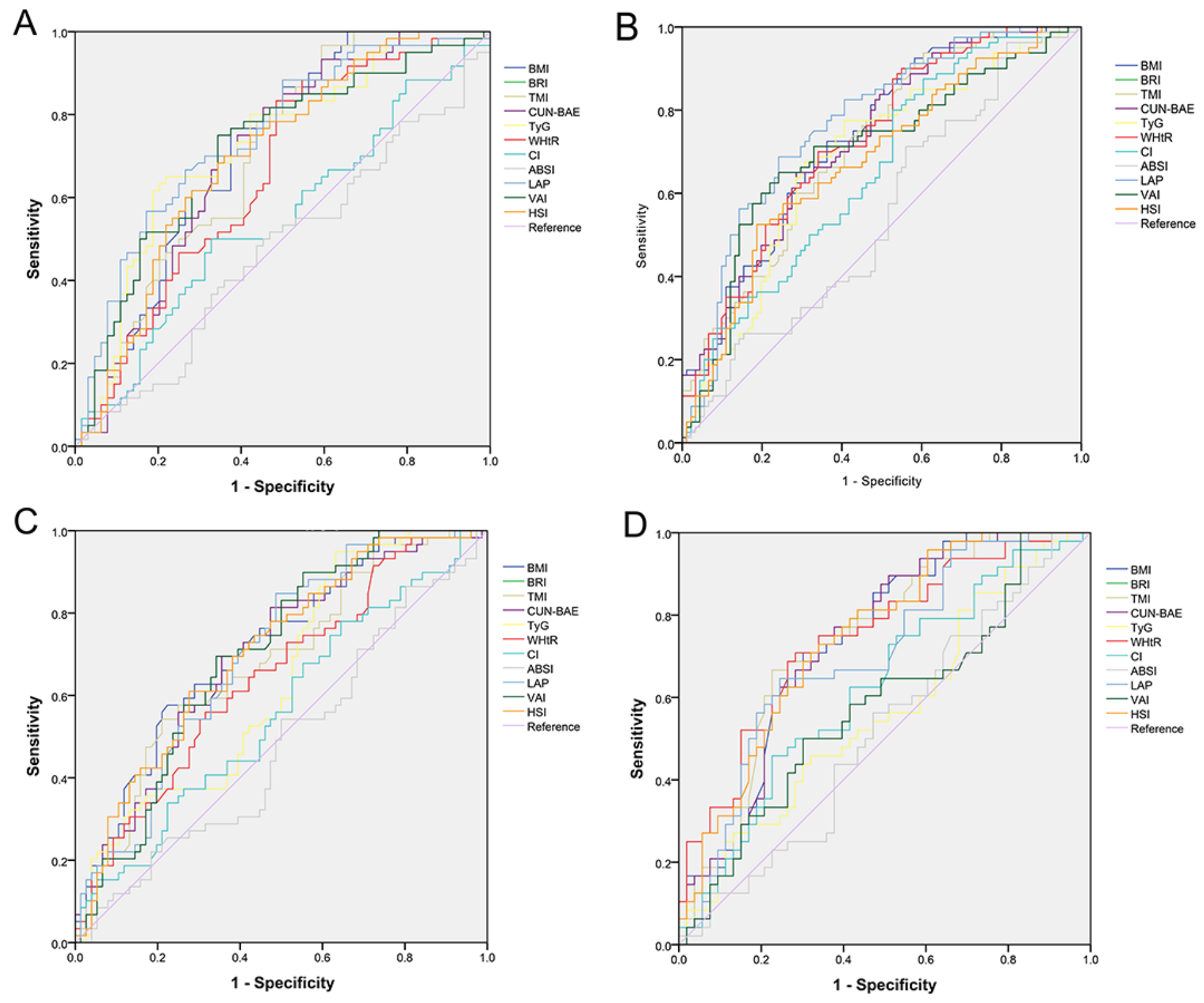

\section{Figure 2}

32q Note『a, males aged 40-69, b, males aged 60 and over, c, females aged 40-69, d, females aged 60 and over

\section{Supplementary Files}

This is a list of supplementary files associated with this preprint. Click to download. 
- 2Table.xlsx

Page 14/14 